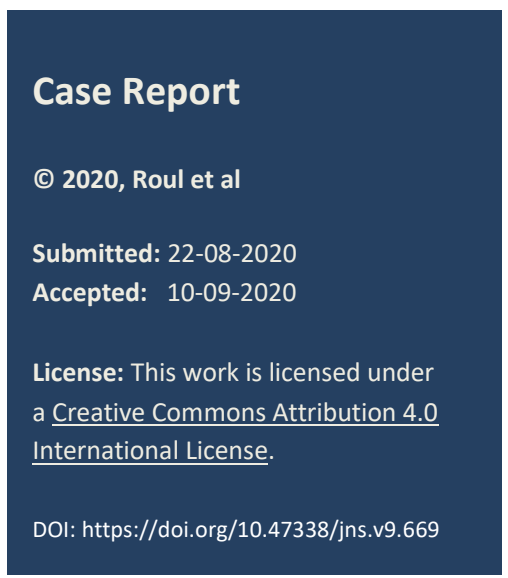

\title{
Imaging pearls of the annular pancreas on antenatal scan and its diagnostic dilemma: A case report
}

Pradeep Kumar Roul, ${ }^{1}$ Ashish Kaushik, ${ }^{1}$ Manish Kumar Gupta, ${ }^{2}$ Poonam Sherwani, ${ }^{1}$ *

1 Department of Radiodiagnosis, All India Institute of Medical Sciences, Rishikesh

2 Department of Pediatric Surgery, All India Institute of Medical Sciences, Rishikesh

Correspondence*: Dr. Poonam Sherwani. DNB, EDIR, Fellow Pediatric Radiology, Department of

Radiodiagnosis, All India Institute of Medical Sciences, Rishikesh, E-mail: sherwanipoonam@gmail.com

\begin{abstract}
KEYWORDS
Annular pancreas,

Duodenal obstruction,

Double bubble sign,

Hyperechogenic band

\section{ABSTRACT}

Background: Annular pancreas is an uncommon cause of duodenal obstruction and rarely causes complete duodenal obstruction. Due to its rarity of identification in the antenatal period and overlapping imaging features with other causes of duodenal obstruction; it is often misdiagnosed.

Case presentation: A 33-year-old primigravida came for routine antenatal ultrasonography at 28 weeks and 4 days of gestational age. On antenatal ultrasonography, dilated duodenum and stomach were seen giving a double bubble sign and a hyperechoic band surrounding the duodenum. Associated polyhydramnios was also present. Fetal MRI was also done. Postpartum ultrasonography demonstrated pancreatic tissue surrounding the duodenum. The upper gastrointestinal contrast study showed a non-passage of contrast beyond the second part of the duodenum. Due to symptoms of obstruction, the neonate was operated on, and the underlying cause was found to be the annular pancreas.

Conclusion: Diagnosis of duodenal obstruction due to the annular pancreas is very important in the antenatal period to plan for appropriate therapeutic interventions after birth. A double bubble sign is seen due to various etiologies however the presence of a hyperechoic band in the antenatal ultrasound may point to the diagnosis of the annular pancreas.
\end{abstract}

\section{INTRODUCTION}

Annular pancreas is a rare congenital malformation characterized by a band or ring of pancreatic tissue that completely or partially encases the second part of the duodenum, resulting in varying degrees of duodenal obstruction.[1] The pancreatic tissue usually spans the second part of the duodenum, partly or completely, close to the papilla or ampulla of Vater, and rarely elsewhere.[2] Symptoms and time of presentation usually depend upon the degree of obstruction caused by the pancreatic tissue or associated duodenal atresia/stenosis.[3]

More importantly, life-threatening duodenal obstruction or atresia are associated with more than $40 \%$ of annular pancreas cases.[4] Thus, if diagnosed antenatally, it represents a valuable predictor for future gastrointestinal emergencies and helpful in further management after birth.

\section{CASE REPORT}

A 33-year-old Indian primigravida with a history of hypothyroidism and $\mathrm{Rh}$ isoimmunization came for routine antenatal ultrasonography at 28 weeks and 4 days of gestation. Her triplet or triple test was negative. In her previous ultrasonography, done at 12 weeks, fetal growth parameters were lagging behind LMP [last menstrual period] by 10 days. The present ultrasound showed mild polyhydramnios with an amniotic fluid index of 28, a single umbilical artery, preaxial polydactyly, and fetal growth retardation. The stomach and duodenum were dilated leading to a double bubble sign with a hyperechogenic band surrounding the fetal duodenum (Fig.1). Differential diagnosis of duodenal obstruction either due to annular pancreas, duodenal atresia/stenosis, or malrotation were given. A maternal right ovarian cyst $(8.1 \times 5.4 \mathrm{~cm})$ was also documented. The fetal amniotic karyotype was normal, 46 XX. Fetal ECHO was also normal. Fetal MRI performed demonstrated 
dilated stomach and the duodenum giving double bubble sign (Fig.2).

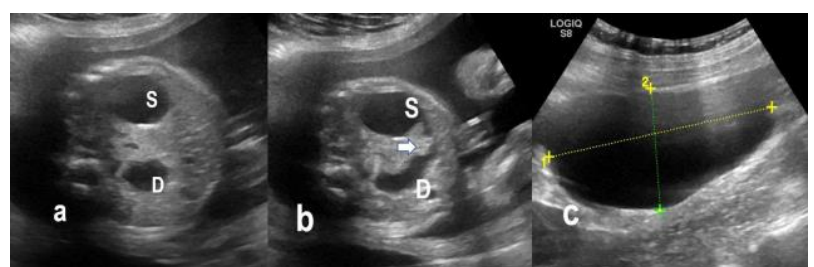

Figure 1: a) Antenatal Ultrasonography at 28 weeks 4 days showing dilated stomach (S) and duodenum (D) giving the Double-bubble sign. b) Hyperechoic band around duodenum (arrow). c) Showing the maternal right ovarian cyst.

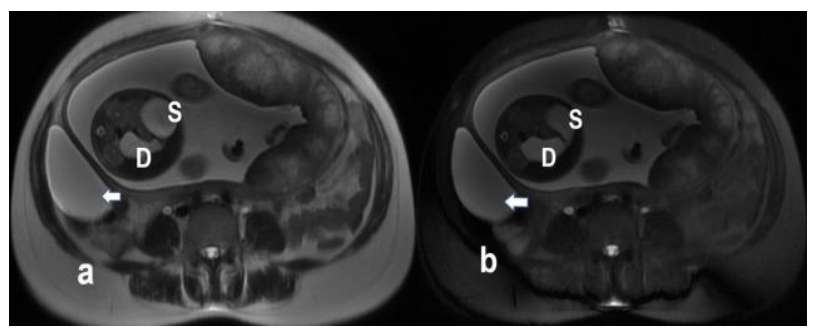

Figure 2: Axial single-shot fast spin-echo with breath-hold (a) and fat-saturated (b) MR images confirming the ultra-sound findings. Dilated fetal stomach (S) and duodenum (D) were seen. Maternal right ovarian cyst showed benign features with no evidence of septation or nodule (arrow).

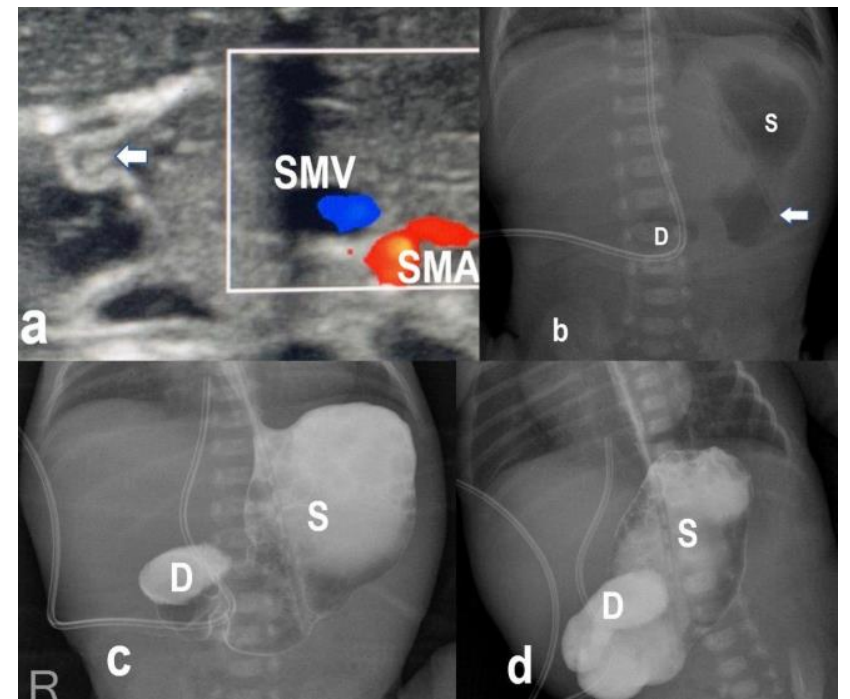

Figure 3: a) Postnatal transverse ultrasonography with color doppler study showing dilated stomach and hyperechoic band around the duodenum (arrow). The normal relationship of superior mesenteric vessels was noted on color doppler ultrasound. b) Frontal radiograph of the abdomen showing distended air-filled stomach (S) and proximal duodenum (D) giving the Double-bubble sign. A feeding tube was also seen with a tip in the stomach (arrow). c-d) Upper GI contrast study spot images AP (c) and right anterior oblique (d) showed distended stomach, D1, and proximal D2 segments of the duodenum. No passage of contrast was seen beyond the 2 nd part of the duodenum.

Due to gestational hypertension and absent end-diastolic flow in the uterine artery, elective lower segment uterine cesarean section was performed at 32 week and 3 days. A small for gestational age female baby was delivered. Cystectomy was also done as part of the procedure and the cyst was benign on histopathology. Ultrasonography done in the immediate postnatal period was consistent with antenatal ultrasonography (Fig.3). The upper gastrointestinal contrast study showed a non-passage of contrast beyond the second part of the duodenum (Fig.3). As annular pancreas rarely causes complete duodenal obstruction, therefore our provisional diagnosis was more inclined to the duodenal atresia rather than the annular pancreas.

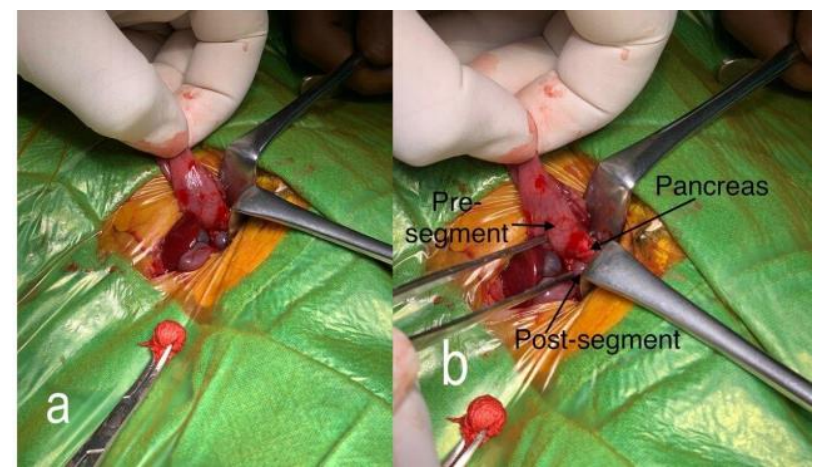

Figure 4a and 4b: Intraoperative photographs showing annular pancreas.

A laparotomy was performed on the second day of the life and the annular pancreas was found causing the duodenal obstruction (Fig.4). A side-to-side duodenoduodenal anastomosis was performed with an uneventful postoperative period.

\section{DISCUSSION}

As most of the cases of the annular pancreas are asymptomatic, no systematic antenatal or postnatal standard screening is practiced. Thus, the real incidence of the annular pancreas is not known. During embryogenesis, the pancreas develops from dorsal and ventral pancreatic buds between the fifth and seventh gestational weeks. In normal development, these two pancreatic buds rotate along with the rotation of the intestine. During that period, the duodenum rotates from left to right, the ventral pancreatic bud typically migrates posteriorly and inferiorly, merging with the more caudal portion of the pancreatic head and the uncinate process, and the dorsal bud develops into the body and tail of the pancreas.[1] The annular pancreas is due to the failure of the ventral bud to rotate, resulting in the incarceration of the duodenum.[5]

Antenatal diagnosis of the annular pancreas is suspected in case of significant duodenal obstruction leading to the double-bubble sign. The distended stomach and the duodenum are seen on the left and center, just to the right of the midline, of the fetal abdomen. Both these dilatations are seen in the prestenotic segment. Double-bubble sign is not specific for the annular pancreas and can be seen with various other conditions like duodenal atresia, tissue bands, and intestine duplications. The ultrasonographic findings of duodenal obstruction are 
usually detected during the second trimester, although two previous studies have described this finding in 1 st trimester.[6,7] It is of utmost importance to identify the underlying cause of duodenal obstruction on antenatal scans, as it helps to define the prognosis and appropriate management after birth.

In our case, a hyperechoic band completely surrounding the duodenum was identified on antenatal as well as postnatal ultrasound scans. Like our case, hyperechoic bands around the duodenum with the double bubble sign secondary to the annular pancreas were reported by a few other authors.[3,8-10] But, we believe the presence of a hyperechoic band in conjunction with a double bubble sign is pathognomonic of the annular pancreas with duodenal obstruction. But this needs validation by further research.

The prenatal diagnosis of the annular pancreas is extremely rare. It is also associated with other abnormalities, like polyhydramnios, Down syndrome, intestinal malrotation, gallbladder agenesis, and

\section{REFERENCES}

1. Schmidt MK, Osvaldt AB, Fraga JCS, Takamatu EE, Fernandes CLS dos S, Rohde L. [Anular pancreaspancreatic resection or duodenal by-pass]. Rev Assoc Med Bras. 2004; 50:74-8.

2. Rinnab L, Schulz K, Siech M. [A rare localization of annular pancreas at the pars horizontalis duodeni-case report]. Zentralbl Chir. 2004; 129:513-6.

3. Dankovcik R, Jirasek E, Kucera E. Prenatal diagnosis of annular pancreas: Reliability of the double bubble sign with periduodenal hyperechogenic band. Fetal Diagn Ther. 2008; 24:483-90.

4. Vijayaraghavan SB. Sonography of pancreatic ductal anatomic characteristics in annular pancreas. J ultrasound Med. 2002; 21:1315-8.

5. Sandrasegaran K, Patel A, Fogel EL, Zyromski NJ, Pitt HA. Annular pancreas in adults. AJR Am J Roentgenol. 2009; 193:455-60.
CHARGE syndrome.[8-10] In our case, there was mild polyhydramnios, single umbilical artery, preaxial polydactyly, and annular pancreas. Therefore, associated anomalies of the annular pancreas must be looked for better management of newborn babies.

In conclusion, the presence of a hyperechoic band in conjunction with a double bubble sign may be pathognomonic of the annular pancreas and should be looked for. Also, there is a need for future studies to prove our hypothesis.

Acknowledgements: Nil

Conflict of Interest: None declared

Source of Support: Nil

Consent to Publication: Author(s) declared taking informed written consent for the publication of clinical photographs/material (if any used), from the legal guardian of the patient with an understanding that every effort will be made to conceal the identity of the patient, however it cannot be guaranteed.

Author Contributions: Author(s) declared to fulfill authorship criteria as devised by ICMJE and approved the final version. Authorship declaration form indicating individual contribution, submitted by the author(s), is available with the editorial office.

6. Tsukerman GL, Krapiva GA, Kirillova IA. Firsttrimester diagnosis of duodenal stenosis associated with oesophageal atresia. Prenat Diagn. 1993; 13:3716.

7. Petrikovsky BM. First-trimester diagnosis of duodenal atresia. Am J Obstet Gynecol. 1994; 171:569-70.

8. Weiss H, Sherer DM, Manning FA. Ultrasonography of fetal annular pancreas. Obstet Gynecol. 1999; 94:852.

9. Mitchell J, Punthakee Z, Lo B, Bernard C, Chong K, Newman C, et al. Neonatal diabetes, with hypoplastic pancreas, intestinal atresia and gall bladder hypoplasia: search for the aetiology of a new autosomal recessive syndrome. Diabetologia. 2004; 47:2160-7.

10. Maier M, Wiesner W, Mengiardi B. Annular pancreas and agenesis of the dorsal pancreas in a patient with polysplenia syndrome. AJR Am J Roentgenol. 2007; 188:W150-3. 